\title{
EXISTENCE OF WEAK SOLUTIONS FOR THE SCHRÖDINGER EQUATION AND ITS APPLICATION
}

\author{
Jinjin Huang \\ Zhoukou Normal University, School of Mathematics and Statistics \\ Zhoukou 466001, P. R. China; huangjinjin@zknu.edu.cn
}

\begin{abstract}
In this paper, we are concerned with the existence of weak solutions for the Schrödinger equation with sign-changing potential in a smooth cone. For solving the Dirichlet boundary-value problem with respect to the Schrödinger operator, we prove the existence of at least one weak solution using changes of Schrödingerean harmonic measure, the energy estimate method and refined inequality technique. Due to the fact that the nonlinearity is allowed to change sign in our formulation, and the novelty of the boundary conditions, these results are new for discrete and arbitrary time scales. As an application, concentration results are also investigated.
\end{abstract}

\section{Introduction and main results}

In this paper, we denote the set $\mathbf{R}_{+} \times \Omega$ with the domain $\Omega$ on $\mathbf{S}^{n-1}$ by $C_{n}(\Omega)$, where $\mathbf{S}^{n-1}$ denotes the unit sphere in $\mathbf{R}^{n}(n \geq 2)$. And we call it a cone. As in $[7,17]$, we also consider the following Schödinger equation

$$
-\triangle u+a(P) u(P)=0,
$$

where $P \in C_{n}(\Omega)$ and $a$ is a nonnegative radical potential satisfying certain conditions. Fractional Laplacian can be understood as the infinitesimal generator of a stable Lèvy diffusion process, and appears in anomalous diffusions in plasmas, flames propagation and chemical reactions in liquids, population dynamics, geographical fluid dynamics, and American options in finance. Please see [1, 3, 20] for more physical background.

In [19], when $a$ is not a radical potential, using variational method, the authors studied the existences and multiplicity results for the following critical Schödinger equation

$$
(-\triangle)^{s} u+a(P) u(P)=0
$$

where $P \in C_{n}(\Omega), 0<s \leq 1$ and the potential $a$ satisfies the following condition

$$
0<\inf _{P=(r, \Theta) \in C_{n}(\Omega)} a(P)<\lim _{r \rightarrow \infty} a(P)=a_{\infty}<\infty .
$$

Invoking Ljusternick-schnirelmann category and Morse theory (see [6]), we investigate the existences and multiplicity results for the following problem

$$
\epsilon^{2 s}(-\triangle)^{s} u+a(P) u(P)=f(u),
$$

where $P \in C_{n}(\Omega)$. They required that $a$ has a positive lower bound. In [4], the authors studied (4) via penalization method. They needed $a$ has a positive lower

https://doi.org/10.5186/aasfm.2019.4452

2010 Mathematics Subject Classification: Primary 35J05, 35J10, 35C15.

Key words: Schödinger equations, sign-changing potential, cone. 
bound and the following local condition

$$
0<c=\inf _{P \in \Lambda} a(P)<\inf _{P \in \partial \Lambda} a(P),
$$

where $\Lambda$ is open bounded set of $C_{n}(\Omega)$. In [11], the author considered the Schödinger equation by Lagrange multiplier method when $a$ has a positive lower bound and

$$
\lim _{r \rightarrow \infty} a(P)=\infty \text {. }
$$

When $\epsilon \rightarrow 0$, (4) is equal to the following Schödinger equation

$$
(-\triangle)^{s} u+\lambda a(P) v=f(P, u)
$$

where $P \in C_{n}(\Omega)$. However, for

$$
f(P, u)=V(P)|u|^{p-2} u+W(P)|u|^{q-2} u,
$$

where $2<q<p<2_{s}^{*}$, the case is different. Especially, $W$ is allowed to be signchanging or negative.

In the present paper, our aim is to investigate, $a$ is sign-changing, $\lambda$ is large, the existences and concentration results for the Schödinger equation. More precisely, we are devoted to study the following problem

$$
(-\triangle)^{s} u+a(P) u \lambda+u a_{0}(P)=|u|^{p-2} u V(P)+W(P)|u|^{q-2} u,
$$

where $P \in C_{n}(\Omega), \lambda$ is large enough and $2<q<p<2_{s}^{*}$.

It is well known that there exists no results in literatures on (5) if $a$ is signchanging.

Throughout this paper, we always make the following assumptions:

(I) $a_{0} \in L^{\infty}\left(C_{n}(\Omega)\right)$ and $a_{0}:=\inf _{x \in \mathrm{R}^{N}} a_{0}(P)>0$.

(II) $a \in C\left(C_{n}(\Omega), \mathbf{R}\right)$ and $a$ is bounded from below.

(III) The set $\left\{P \in C_{n}(\Omega): a(P)<b\right\}$ has finite measure and is nonempty, where $b$ is a positive constant.

(IV) Define $\Omega=\operatorname{int} a^{-1}(0)$. Then it is easy to see that it has smooth boundary and is nonempty. Meanwhile, $\bar{\Omega}=a^{-1}(0)$.

(V) $V(P) \in L^{\infty}\left(C_{n}(\Omega)\right), V_{0}:=\inf _{P \in C_{n}(\Omega)} V(P)>0$, and there exists a positive constant $C(\lambda)$ satisfying $\left\|V_{0}\right\|_{L} \infty \leq C(\lambda)$.

(VI) $W(P) \in L^{\infty}\left(C_{n}(\Omega)\right)$. $W$ is sign-changing or negative and

$$
\left\{P \in C_{n}(\Omega): W(P) \geq 0\right\}
$$

has finite measure.

Theorem 1.1. If a changes its sign, then (5) has at least a weak solution $u_{\lambda}$.

Theorem 1.2. If $a(P) \geq 0$, then (5) has at least a weak solution $u_{\lambda}$. Furthermore, $u_{\lambda} \rightarrow \bar{u}$ in $H^{s}\left(C_{n}(\Omega)\right)$ as $\lambda \rightarrow \infty$, where $\bar{u}$ is a weak solution of

$$
\begin{cases}(-\triangle)^{s} u+a_{0}(P) u=|u|^{p-2} u V(P)+W(P)|u|^{q-2} u, & \text { in } \Omega, \\ u=0, & \text { on } \partial \Omega .\end{cases}
$$

Remark 1.1. If $W$ satisfies $W(P) \in L^{\infty}(\mathbf{R}), W_{0}:=\inf _{x \in C_{n}(\Omega)} W(P)>0$. Theorems 1.1 and 1.2 remain true. In fact, we only need to modify our proofs mildly.

Remark 1.2. a may be coercive or not coercive.

In [13], $a(P)$ with $\left(V_{1}\right)-(\mathrm{IV})$ is first referred as the steep well potential in the study of nonlinear Schrödinger equation. For more details, the reader is referred to the papers $[9,10,23,22]$, to the book [15] and to the excellent article [18]. Recently, 
Properties of weak solutions for the stationary Schrödinger equation with radical potential in a smooth cone and cylinder were studied in $[13,12,11]$.

We should point out that we need to reestablish the eigenvalue problem of fractional Schrödinger. And during the research, there are some technical difficulties to recover compact condition. $c_{\lambda}$ is uniformly bounded about $\lambda$ is used to recover compactness, so we need a elaborate estimate since it is different from Schrödinger equations. It is very interesting that the initial thought in our present paper is to study concentration results when $V$ is sign-changing, but we fail since it seems to need $a(P) \geq 0$ in (5) to obtain $\left\{u_{\lambda}\right\}$ is bounded in $E$.

Denote Lebesgue measure of $A$ by $|A| \cdot|\cdot|_{p}$ is the usual norm of $L^{p}\left(C_{n}(\Omega)\right)$. $C$ and $C_{i}$ are positive constants, which may be different from line to line. At first, we recall $(-\triangle)^{s}: S \rightarrow L^{2}\left(C_{n}(\Omega)\right)$ is defined by (see [24])

$$
(-\triangle)^{s} u(P)=\int_{C_{n}(\Omega)} \frac{u(P)-u(Q)}{|P-Q|^{n+2 s}} d Q,
$$

where $S$ is be the Schwartz space of rapidly decaying $C^{\infty}$ functions in $C_{n}(\Omega)$. The Sobolev space $H^{S}\left(C_{n}(\Omega)\right)$ is defined as

$$
H^{s}\left(C_{n}(\Omega)\right)=\left\{u \in L^{2}\left(C_{n}(\Omega)\right) \frac{|u(P)-u(Q)|^{2}}{|P-Q|^{n+2 s}} \in L^{1}\left(C_{n}(\Omega) \times C_{n}(\Omega)\right)\right\}
$$

endowed

$$
(u, v)_{H^{s}}=\int_{C_{n}(\Omega)}\left((-\triangle)^{\frac{s}{2}} u(-\triangle)^{\frac{s}{2}} v+u v\right) d P
$$

and norm

$$
\|u\|_{H^{s}}^{2}=\int_{C_{n}(\Omega)}\left(\left|(-\triangle)^{s} 2 u\right|^{2}+u^{2}\right) d P
$$

Let

$$
E=\left\{u \in H^{s}\left(C_{n}(\Omega)\right): \int_{C_{n}(\Omega)} a^{+}(P) u^{2} d P<\infty\right\},
$$

be equipped with

$$
(u, v)=\int_{C_{n}(\Omega)}\left((-\triangle)^{\frac{s}{2}} u(-\triangle)^{\frac{s}{2}} v+a^{+}(P) u v\right) d P
$$

and

Fixed $\lambda>0$,

$$
\|u\|=(u, u)^{\frac{1}{2}}
$$

$$
(u, v)_{\lambda}=\int_{1 C_{n}(\Omega)}\left((-\triangle)^{\frac{s}{2}} u(-\triangle)^{\frac{s}{2}} v+\lambda a^{+}(P) u v\right) d P, \quad\|u\|_{\lambda}=(u, u)_{\lambda}^{\frac{1}{2}}
$$

Set $E_{\lambda}=\left(E,\|\cdot\|_{\lambda}\right)$. Clearly, $\|u\| \leq\|u\|_{\lambda}$.

Lemma 1.1. $E \hookrightarrow H^{S}\left(C_{n}(\Omega)\right) \hookrightarrow L^{t}\left(C_{n}(\Omega)\right)$ for $t \in\left[2,2_{s^{*}}\right]$ and $E \hookrightarrow L_{\text {loc }}^{t}\left(C_{n}(\Omega)\right)$ for $t \in\left[1,2_{s} *\right)$ is compact.

Proof. Noting that $(I I I)$, it holds that $a^{+}(P) \not \equiv 0$. Denote

$$
\begin{aligned}
& A(R):=\left\{P=(r, \Theta) \in C_{n}(\Omega), \quad R<r, b \leq a^{+}(P)\right\}, \\
& B(R):=\left\{P=(r, \Theta) \in C_{n}(\Omega), \quad R<r, b>a^{+}(P)\right\} .
\end{aligned}
$$

Obviously,

$$
\int_{A(R)} u^{2} d P \leq \frac{1}{b} \int_{C_{n}(\Omega)} u^{2} d P
$$


Since Hölder inequality and (4) of [14], jointly with $|B(R)|<\infty$, we obtain

$$
\int_{B(R)} u^{2} d P \leq C \int_{C_{n}(\Omega)}\left|(-\triangle)^{\frac{s}{2}} u\right|^{2} d P
$$

Similarly,

$$
\int_{C_{n}(\Omega ;(0, R])} u^{2} d P \leq C \int_{C_{n}(\Omega)}\left|(-\triangle)^{\frac{s}{2}} u\right|^{2} d P .
$$

Therefore $E \hookrightarrow H^{S}\left(C_{n}(\Omega)\right)$. It is well known that $H^{S}\left(C_{n}(\Omega)\right) \hookrightarrow L^{p}\left(C_{n}(\Omega)\right)$ is continuous. The compact embedding follows from Rellich compactness theorem.

The functional of (5) can be written as follows

$$
\begin{aligned}
I_{\lambda}(u)= & \frac{1}{2} \int_{C_{n}(\Omega)}\left(\left|(-\triangle)^{\frac{s}{2}} u\right|^{2}+\lambda a(P) u^{2}+a_{0}(P) u^{2}\right) d P \\
& -\frac{1}{p} \int_{C_{n}(\Omega)} V(P)|u|^{p} d P-\frac{1}{q} \int_{C_{n}(\Omega)} W(P)|u|^{p} d P .
\end{aligned}
$$

Clearly, using Lemma 1.1 and Lebesgue dominated theorem, $I_{\lambda}$ is $C^{1}$ on $E$. $u \in H^{S}\left(C_{n}(\Omega)\right)$ is a weak solution of $(5)$ if and only if $I_{\lambda}^{\prime}(u)=0$. Since $a$ is signchanging, the quadric form

$$
a_{\lambda}(u, u) \int_{C_{n}(\Omega)}\left(\left|(-\triangle)^{\frac{s}{2}} u\right|^{2}+\lambda a(P) u^{2}\right) d P
$$

is indefinite. Denote

$$
D:=\left\{u \in E: \operatorname{supp} u \subset a^{-1}([0, \infty])\right\}
$$

and

$$
F=\bar{D}^{\|\cdot\|_{\lambda}}=\overline{\left\{u \in E: \operatorname{supp} u \subset a^{-1}([0, \infty])\right\}^{\| \cdot}} \|_{\lambda},
$$

which means that $F$ is closure of $D$ under the norm $\|\cdot\|_{\lambda}$. According to [16, Theorem 12.4], there exists a closed subspace $M$ such that $E_{\lambda}=F \oplus M$. If $a(P) \geq 0$, then $E=F$.

Consider the bilinear form

$$
a_{\lambda}(u, v)=\int_{C_{n}(\Omega)}\left((-\triangle)^{\frac{s}{2}} u(-\triangle)^{\frac{s}{2}} v+\lambda a(P) u v\right) d P,
$$

and the eigenvalue problem

$$
(-\triangle)^{s} u+\lambda a^{+}(P) u=\alpha \lambda V^{-}(P) u,
$$

where $u \in M$.

Let $A:=\operatorname{supp} a^{-}$. Noting that $(I I I)$, it holds $|A|<\infty$. It is easy to check that there is $C>0$, such that for any $u \in M$,

$$
\begin{array}{r}
\int_{C_{n}(\Omega)}\left(\left|(-\triangle)^{\frac{s}{2}} u\right|^{2}+\lambda a^{+}(P) u^{2}\right) d P \geq C \int_{A} \lambda a^{-}(P) u^{2} d P, \\
W(u):=\int_{C_{n}(\Omega)}\left(\left|(-\triangle)^{\frac{s}{2}} u\right|^{2}+\lambda a^{+}(P) u^{2}\right) d P .
\end{array}
$$

Lemma 1.2.

$$
\alpha_{1}(\lambda)=\inf _{0 \neq u \in M} \frac{W(u)}{\int_{A} \lambda a^{-}(P) u^{2} d P}>0
$$

is achieved and $\alpha_{1}(\lambda)$ is the smallest eigenvalue of (8). 
Proof. The proof is standard (see [8, p. 74]). The difference is that $M$ is not the whole space $E_{\lambda}$. But $M$ is convex closed set. Let $\left\{u_{k}\right\} \subset M$ be a minimizing sequence. In view of $M$ is convex closed set, there exists $u \in M$ such that $u_{k} \rightarrow u$ in $E_{\lambda}$. Combining with compact embedding Lemma 1.1, similarly to [8], we obtain the desired conclusions.

Similarly to $\left[8\right.$, p. 80] (see also $\left[5\right.$, p. 13]), $\lambda>0$, we can define $\alpha_{j}(\lambda), j=2,3, \ldots$. The following results are essentially known.

Proposition 1. Each eigen-subspace is finite dimensional.

Proposition 2. Fixed $\lambda>0, \alpha_{j}(\lambda) \stackrel{j \rightarrow \infty}{\longrightarrow} 0$.

Proposition 3. The eigenfunctions for different eigenvalue are orthogonal in $E_{\lambda}$.

Lemma 1.3. Fixed $j, a_{j}(\lambda) \stackrel{\lambda \rightarrow \infty}{\longrightarrow} 0$.

Proof. Here we may always assume $j \geq 2$. Let $u_{i} \in M$ be the corresponding eigenfunctions of $\left.\alpha_{i}(\lambda), i=1,2, \ldots, j-l\right)$. Denote $\mathrm{V}_{j-1}=\operatorname{span}\left\{u_{i}, i=1,2, \ldots, j-\right.$ 1\}. $V_{j-1}^{\perp}$ denotes the orthogonal complement of $\mathrm{V}_{j-1}$ in $L^{2}$. Let $u \in C_{0}^{\infty}\left(C_{n}(\Omega)\right)$ such that $\operatorname{supp} u \subset \operatorname{supp} a^{-}, \operatorname{supp} u \cap \operatorname{supp} u_{i}=\emptyset, 1 \leq i \leq j-1$. Thus it holds that

$$
\alpha_{j}(\lambda) \leq \frac{\int_{C_{n}(\Omega)}|\nabla u|^{2}}{\lambda \int_{C_{n}(\Omega)} a^{-}(P) u^{2}} \rightarrow 0 \quad \text { as } \lambda \rightarrow \infty .
$$

It follows that there exists $\Lambda>0$ satisfying

$$
\hat{E}_{\lambda}:=\operatorname{span}\left\{e_{j} \alpha_{j}(\lambda) \leq 1\right\}
$$

when $\lambda>\Lambda$ from Lemma 1.3 is not empty and $a_{\lambda}(u, u)$ is negative semidefinite in $\hat{E}_{\lambda}$, where $e_{j}$ is the corresponding eigenfunctions of $\alpha_{j}(\lambda)$. Set $E_{\lambda}^{+}:=\operatorname{span}\left\{e_{j} \alpha_{j}(\lambda)>1\right\}$. In the light of Propositions 1 and $3, E_{\lambda}=M \oplus F=\hat{E}_{\lambda} \oplus E_{\lambda}^{+} \oplus F$ and $\operatorname{dim} E_{\lambda}<\infty$.

\section{Proof of Theorem 1.1}

We shall prove that $I_{\lambda}$ satisfies linking geometric construction of [21, Theorem 2.1.2].

Lemma 2.1. For each $\lambda>\Lambda$, there exist $\rho_{\lambda}>0$ and $\kappa_{\lambda}>0$ such that

$$
I_{\lambda}(u) \geq \kappa_{\lambda}
$$

for all $u \in E_{\lambda}^{+} \oplus F$ with $\|u\|_{\lambda}=\rho_{\lambda}$.

Proof. By the definition of $E_{\lambda}^{+}$, there is $\delta_{\lambda}>0$ satsifying

$$
a_{\lambda}(u, u) \geq \delta_{\lambda}\|u\|_{\lambda}^{2}
$$

for $u \in E_{\lambda}^{+}$and

$$
a_{\lambda}(u, u)=\|u\|_{\lambda}^{2},
$$

where $u \in F$. Thus for $u=v+w \in E_{\lambda}^{+} \oplus F$, invoking Sobolev embedding, we get

$$
\begin{aligned}
I_{\lambda}(u)= & \frac{1}{2} a_{\lambda}(v, v)+\frac{1}{2} a_{\lambda}(w, w)+\frac{1}{2} \int_{C_{n}(\Omega)} a_{0}(P) u^{2} d P \\
& -\frac{1}{p} \int_{C_{n}(\Omega)} V(P)|u|^{p} d P-\frac{1}{q} \int_{C_{n}(\Omega)} W(P)|u|^{q} d P \\
\geq & \frac{1}{2} \min \left\{\delta_{\lambda}, 1\right\}\|u\|_{\lambda}^{2}-C_{1}\|u\|_{\lambda}^{p}-C_{2}\|u\|_{\lambda}^{q} .
\end{aligned}
$$


Taking $\epsilon>0, \rho_{\lambda}>0$ and $\kappa_{\lambda}>0$ small enough, we have the desired conclusion.

By (IV), we take $e_{0} \in C_{0}^{\infty}(\Omega)$, then $e_{0} \in F$.

Lemma 2.2. For each $\lambda>\Lambda$, there exists $R_{\lambda}>0$ such that

$$
\sup _{u \in \partial Q} I_{\lambda}(u)<\kappa_{\lambda}
$$

where

$$
Q=\left\{u=v+t e_{0}: v \in \hat{E}_{\lambda}, t \geq 0,\|u\|_{\lambda} \leq R_{\lambda}\right\} .
$$

Proof. In view of (V) and (VI), there exist $C_{1}>0, C_{2}>0$ such that

$$
\frac{1}{p} V(P)|u|^{p}+\frac{1}{q} W(P)|u|^{q} \geq C_{1}|u|^{p}-C_{2}|t|^{q} .
$$

If $u=v+w \in \hat{E}_{\lambda} \oplus R e_{0}$, noting that

$$
a_{\lambda}(u, u)=a_{\lambda}(v, v)+a_{\lambda}(w, w) \leq\|u\|_{\lambda}^{2},
$$

jointly with all the norms on finite dimensional space are equivalent and $q<p$, we obtain

$$
I_{\lambda}(u) \leq C\|u\|_{\lambda}^{2}+C_{3}\|u\|_{\lambda}^{q}-C_{4}\|u\|_{\lambda}^{p} \stackrel{\|u\|_{\lambda} \rightarrow \infty}{\longrightarrow}-\infty .
$$

Therefore, there exists $R_{\lambda}>0$ such that $I_{\lambda}(u) \leq 0$ for $u \in \hat{E}_{\lambda} \oplus R e_{0}$ with $\|u\|_{\lambda}=R_{\lambda_{\wedge}}$. If $u \in \hat{E}_{\lambda}$, then we have

$$
I_{\lambda}(u) \leq \frac{1}{2} \int_{C_{n}(\Omega)} a_{0}(P) u^{2} d P+C_{3}\|u\|_{\lambda}^{q}-C_{4}\|u\|_{\lambda}^{p} .
$$

So we also obtain the conclusion.

According to Lemmas 2.1 and $2.2, I_{\lambda}$ has a sequence $\left\{u_{n}\right\} \subset E_{\lambda}$, i.e.,

$$
I_{\lambda}\left(u_{n}\right) \rightarrow c,\left(1+\left\|u_{n}\right\|_{\lambda}\right) I_{\lambda}^{\prime}\left(u_{n}\right) \rightarrow 0 \quad \text { in } E_{\lambda}^{*} .
$$

Lemma 2.3. $\left\{u_{n}\right\}$ is bounded (dependent on $\lambda$ ) in $E_{\lambda}$.

Proof. For $n$ large enough, we have

$$
\begin{aligned}
I_{\lambda}\left(u_{n}\right)-\frac{1}{q}\left\langle I_{\lambda}^{\prime}\left(u_{n}\right), u_{n}\right\rangle= & \left(\frac{1}{2}-\frac{1}{q}\right)\left\|u_{n}\right\|_{\lambda}^{2}-\left(\frac{1}{2}-\frac{1}{q}\right) \int_{C_{n}(\Omega)} \lambda a^{-}(P) u_{n}^{2} d P \\
& +\left(\frac{1}{2}-\frac{1}{q}\right) \int_{C_{n}(\Omega)} a_{0}(P) u_{n}^{2} d P \\
& +\left(\frac{1}{q}-\frac{1}{p}\right) \int_{C_{n}(\Omega)} V(P)\left|u_{n}\right|^{p} d P=c+1 .
\end{aligned}
$$

Thus, combining with (II), we get

$$
\left\|u_{n}\right\|_{\lambda}^{2} \leq C_{1} \lambda \int_{C_{n}(\Omega)} u_{n}^{2} d P+C_{2} .
$$

Thus it suffices to prove that $\left\{u_{n}\right\}$ is bounded in $L^{2}\left(C_{n}(\Omega)\right)$. If

$$
\int_{C_{n}(\Omega)} u_{n}^{2} d P \stackrel{n \rightarrow \infty}{\longrightarrow} \infty
$$

Denote $v_{n}=\frac{u_{n}}{\left|u_{n}\right|_{2}}$, then $\left|v_{n}\right|_{2}=1$. By (14), we have

$$
\left\|v_{n}\right\|_{\lambda}^{2}+\int_{C_{n}(\Omega)} a_{0}(P) v_{n}^{2} d P-\int_{C_{n}(\Omega)} \lambda a^{-}(P) v_{n}^{2} d P \leq \frac{C}{\left|u_{n}\right|_{2}^{2}} .
$$


Consequently, $\left\{\left\|v_{n}\right\|_{\lambda}^{2}\right\}$ is bounded and

$$
\int_{C_{n}(\Omega)} a(P) v_{n}^{2} d P \leq \frac{C}{\left|u_{n}\right|_{2}^{2}} \stackrel{n \rightarrow \infty}{\longrightarrow} 0 .
$$

Up to a subsequence we can assume $v_{n} \rightarrow v$ in $E_{\lambda}$. It is easy to obtain $v=0$ from (15) and Fatou's lemma. Thus, we have

$$
\begin{aligned}
\int_{C_{n}(\Omega)} a(P) v_{n}^{2} d P & =\int_{\{x: a(P) \geq b\}} a(P) v_{n}^{2} d P+\int_{\{x: a(P)<b\}} a(P) v_{n}^{2} d P \\
& \geq b\left(1-\int_{\{x: a(P)<b\}} a(P) v_{n}^{2} d P\right)+o_{n}(1) \geq b+o_{n}(1)>0
\end{aligned}
$$

which contracts with (16).

Next lemma will be used in Lemma 2.5 to prove $(C)_{c}$ condition.

Lemma 2.4. $c_{\lambda}$ is uniformly bounded about $\lambda$.

Proof. In the light of [21, Theorem 2.1.2], $c_{\lambda} \in\left[\kappa_{\kappa}, \sup _{u \in Q} I_{\lambda}(u)\right]$. We only need to prove $\sup _{u \in Q} I_{\lambda}(u)$ has a positive bound from above independent of $\lambda$. Set

$$
J_{\lambda}(u):=\frac{1}{2} a_{\lambda}(u, u)+\frac{1}{2} \int_{C_{n}(\Omega)} a_{0}(P) u^{2} d P-C_{1} \int_{\Omega}|u|^{q} d P,
$$

where $u \in E_{\lambda}$.

A simple estimate, $I_{\lambda}(u) \leq J_{\lambda}(u)$. It follows that there exists $r_{\eta}>0$ satisfying

$$
\begin{cases}C_{1}|u|^{q} \geq \frac{1}{2} \eta u^{2}, & |u| \geq r_{\eta}, \\ C_{1}|u|^{q} \leq \frac{1}{2} \eta u^{2}, & |u| \leq r_{\eta},\end{cases}
$$

for any $\eta>0$. Let $u=v+w \in \hat{E}_{\lambda} \oplus R e_{0}$. It holds that

$$
\begin{aligned}
J_{\lambda}(u) \leq & \frac{1}{2} \int_{C_{n}(\Omega)}\left|(-\triangle)^{s} 2 w\right|^{2} d P+\frac{1}{2} \int_{C_{n}(\Omega)} a_{0}(P) u^{2} d P-\frac{1}{2} \eta \int_{\Omega} u^{2} d P \\
& +\int_{\left\{x \in \Omega:|u(P)| \leq r_{\eta}\right\}}\left(\frac{1}{2} \eta u^{2}-C_{1}|u|^{q}\right) d P \\
\leq & \frac{1}{2} \int_{C_{n}(\Omega)}\left|(-\triangle)^{s} 2 w\right|^{2} d P+C\left\|V_{0}\right\|_{L \infty}\|u\|_{\lambda}^{2}-\frac{\eta}{2} \int_{\Omega} u^{2} d P+C(\eta) .
\end{aligned}
$$

In view of $e_{0} \in C_{0}^{\infty}(\Omega)$, we get

$$
\int_{C_{n}(\Omega)}\left|(-\triangle)^{\frac{s}{2}} w\right|^{2} d P=a_{\lambda}(u, w)=\int_{\Omega} u(-\triangle)^{s} w d P \leq\left|(-\triangle)^{s} w\right|_{L^{2}(\Omega)}|u|_{L^{2}(\Omega)} .
$$

Let $\left(\varphi_{k}, \mu_{k}\right)$ be the eigenfunctions and eigenvalues of $-\triangle$ in $\Omega$ with Dirichlet boundary data. According to [2, Lemmas 3.4 and 3.5], it holds

$$
\left\|(-\triangle)^{s} w\right\|_{L^{2}(\Omega)}=\left(\sum_{k=1}^{\infty} a_{k}^{2} \mu_{k}^{s}\right)^{\frac{1}{2}}<\infty,
$$

where

$$
a_{k}=\int_{\Omega} w \varphi_{k} d P
$$


In view of $0<\mu_{1} \leq \mu_{2} \cdots \leq \mu_{k} \cdots$ and $\mu_{k} \rightarrow \infty$ as $k \rightarrow \infty$, we get

$$
\left\|(-\triangle)^{\frac{s}{2}} w\right\|_{L^{2}(\Omega)}=\left(\sum_{k=1}^{\infty} a_{k}^{2} \mu_{k}^{\frac{s}{2}}\right) \frac{1}{2}<\infty \text {. }
$$

So

$$
\left\|(-\triangle)^{s} w\right\|_{L^{2}(\Omega)} \leq C_{0}\left\|(-\triangle)^{\frac{s}{2}} w\right\|_{L^{2}(\Omega)},
$$

where $C_{0}$ is only dependent with $e_{0}$.

Noting that (18)-(20), using Young inequality, it is easy to check

$$
\int_{C_{n}(\Omega)}\left|(-\triangle)^{\frac{s}{2}} w\right|^{2} d P \leq \frac{2}{\eta} C_{0}^{2}\left\|(-\triangle)^{\frac{s}{2}} w\right\|_{L^{2}(\Omega)}^{2}+\frac{\eta}{2}\|u\|_{L^{2}(\Omega)}^{2} .
$$

Let $\eta \geq 4 C_{0}^{2}$. Then

$$
\int_{C_{n}(\Omega)}\left|(-\triangle)^{\frac{s}{2}} w\right|^{2} u \|_{L^{2}(\Omega)}^{2}
$$

If $\left\|V_{0}\right\|_{L} \infty \leq \frac{2}{C R_{\lambda}^{2}}$, according to $(17)$, we have $J_{\lambda}(u) \leq C(\eta)+1$.

Lemma 2.5. Let $M$ be any positive constant. There is $\Lambda=\Lambda(M)>0$ such that $(C)_{c}$ sequence $\left\{u_{n}\right\}$ satisfies $u_{n} \rightarrow u$ in $E_{\lambda}$, where $u$ is a weak critical point of $I_{\lambda}$ or $I_{\lambda}$ satisfies $(C)_{c}$ condition, where $c \leq M$.

Proof. In view of Lemma 2.3, if necessary going to a subsequence, $u_{n} \rightarrow u$ in $E_{\lambda}$. Since $\mathrm{V}$ is sign-changing, we need to divide into two cases:

Case (i). $I_{\lambda}(u)<0$.

Case (ii). $I_{\lambda}(u) \geq 0$.

If (i) holds, then it is easy to see that $u$ is weak and the proof is completed. If (ii) holds, then we will show that $u_{n} \rightarrow u$ in $E_{\lambda}$. Let $v_{n}=u_{n}-u$. By (iii),

$$
\int_{C_{n}(\Omega)} v_{n}^{2} d P=\int_{\{x: a(P) \geq b\}}^{v_{n}^{2}} d P+\int_{\{x: a(P)<b\}}^{v_{n}} d P \leq \frac{1}{\lambda b}\left\|v_{n}\right\|_{\lambda}^{2}+o_{n}(1) .
$$

Thus, it follows from interpolation inequality and Sobolev inequalities that

$$
\left|v_{n}\right|_{p} \leq\left|v_{n}\right|_{2}^{\sigma}\left|v_{n}\right|_{2_{s}^{*}}^{1-\sigma} \leq d\left|v_{n}\right|_{2}^{\sigma}\left|(-\triangle)^{\frac{1}{2}} v_{n}\right|_{2}^{1-\sigma} \leq d(\lambda b)^{-\frac{\sigma}{2}}\left\|v_{n}\right\|_{\lambda}+o_{n}(1),
$$

where $0<\sigma<1, d$ is a constant. We have

$$
I_{\lambda}\left(v_{n}\right)=I_{\lambda}\left(u_{n}\right)-I_{\lambda}(u)+o_{n}(1), \quad I_{\lambda}^{\prime}\left(v_{n}\right)=I_{\lambda}^{\prime}\left(u_{n}\right)+o_{n}(1)
$$

from Brezis-Lieb lemma.

A direct computation, there holds that

$$
\begin{aligned}
\left(\frac{1}{q}-\frac{1}{p}\right) \int_{C_{n}(\Omega)} V(P)\left|v_{n}\right|^{p} d P & \leq I_{\lambda}\left(v_{n}\right)-\frac{1}{q}\left\langle I_{\lambda}^{\prime}\left(v_{n}\right), v_{n}\right\rangle \\
& =c-I_{\lambda}(u)+o_{n}(1) \leq M+o_{n}(1) .
\end{aligned}
$$

Therefore,

$$
\left|v_{n}\right|_{p}^{p} \leq \frac{M p q}{V_{0}(p-q)}+o_{n}(1) .
$$

Noting that condition (VI) and

$$
\int_{C_{n}(\Omega)} \lambda a^{-}(P) v_{n}^{2} d P=o_{n}(1)
$$


it follows from $(22)-(25)$ that

$$
\begin{aligned}
o_{n}(1) & =\left\langle I_{\lambda}^{\prime}\left(v_{n}\right), v_{n}\right\rangle \geq\left\|v_{n}\right\|_{\lambda}^{2}-C_{1}\left|v_{n}\right|_{p}^{p}+o_{n}(1) \\
& \geq\left(1-C_{1}\left(\frac{M p q}{V_{0}(p-q)}\right)^{\frac{\mathrm{p}-2}{\mathrm{p}}} \frac{d^{2}}{(\lambda b)^{\sigma}}\right)\left\|v_{n}\right\|_{\lambda}^{2}+o_{n}(1) .
\end{aligned}
$$

Choosing A large enough, we get $v_{n} \rightarrow 0$ in $E_{\lambda}$.

\section{Proof of Theorem 1.2}

According to our assumptions on Theorem 1.2, we obtain that $I_{\lambda}$ has mountain pass geometry. Invoking [14, Theorem 2.2], $I_{\lambda}$ has a $C_{c}$ sequence. Note that $\hat{E}_{\lambda}=\{0\}$ does not influence the results of Lemmas 2.3 and 2.5. Similarly to Lemmas 2.3 and 2.5, we obtain a weak critical point $u_{\lambda}$ for $I_{\lambda}$ with $I_{\lambda}\left(u_{\lambda}\right) \in\left[\kappa, C_{0}\right]$ for $\lambda$ large enough where $\kappa$ and $C_{0}$ are independent of $\lambda$.

A direct computation, we get

$$
\begin{aligned}
c_{\lambda_{n}}= & I_{\lambda_{n}}\left(u_{n}\right)-\frac{1}{q}\left\langle I_{\lambda_{n}}^{\prime}\left(u_{n}\right), u_{n}\right\rangle \\
= & \left(\frac{1}{2}-\frac{1}{q}\right)\left\|u_{n}\right\|_{\lambda_{n}}^{2}+\left(\frac{1}{2}-\frac{1}{q}\right) \int_{C_{n}(\Omega)} a_{0}(P) u_{n}^{2} d P \\
& +\left(\frac{1}{q}-\frac{1}{p}\right) \int_{1 C_{n}(\Omega)} V(P)\left|u_{n}\right|^{p} d P \geq\left(\frac{1}{2}-\frac{1}{\theta}\right)\left\|u_{n}\right\|_{\lambda_{n}}^{2} .
\end{aligned}
$$

It follows from $(27)$ that $\left\{u_{n}\right\}$ is bounded in $E$. After extracting a subsequence, we may assume $u_{n} \rightarrow \bar{u}$ in $E$. We have

$$
\int_{C_{n}(\Omega)} a(P) \bar{u}^{2} d P \leq \liminf _{n \rightarrow \infty} \int_{C_{n}(\Omega)} a(P) \bar{u}_{n}^{2} d P \leq \liminf _{n \rightarrow \infty} \frac{\left\|u_{n}\right\|_{\lambda_{n}}^{2}}{\lambda_{n}}=0
$$

from Fatou's Lemma.

So $\bar{u}=0$ a.e. in $C_{n}(\Omega) \backslash a^{-1}(0), \bar{u} \in H_{0}^{s}(\Omega)$ by (IV). Since for any $\varphi \in C_{0}^{\infty}(\Omega)$, $\left\langle I_{\lambda_{n}}^{\prime}\left(u_{n}\right), \varphi\right\rangle=0$, it is easy to check $\bar{u}$ is a weak solution of (5). To complete the proof, we only need to prove $u_{n} \rightarrow \bar{u}$ in $E$ and $\bar{u} \neq 0$. The proof is almost standard and we refer the readers to [14, Section 3.5] with slight modification. Here we sketch the proof for the readers' convenience.

Step 1. Using Lions vanishing [21, Lemma 12.1], we have $u_{n} \rightarrow \bar{u}$ in $L^{t}\left(C_{n}(\Omega)\right)$ for $2<t<2_{s}^{*}$.

Step 2. By $\left\langle I_{\lambda_{n}}^{\prime}\left(u_{n}\right), u_{n}\right\rangle=\left\langle I_{\lambda_{n}}^{\prime}\left(u_{n}\right), \hat{u}\right\rangle$ we get $u_{n} \rightarrow \bar{u}$ in $E$.

Step 3. $\bar{u} \neq 0$ is obtained since $u_{n} \neq 0$ and $u_{n} \rightarrow \bar{u}$ in $E$.

Acknowledgment. The author wishes to thank the anonymous referees very much for carefully reading this paper and suggesting many valuable comments.

\section{References}

[1] Baskonus, H. M., T. A. Sulaiman, H. Bulut, and T. Aktürk: Investigations of dark, bright, combined dark-bright optical and other soliton solutions in the complex cubic nonlinear Schrödinger equation with delta-potential. - Superlattices Microstruct. 115, 2018, 19-29.

[2] Brändle, C., E. Colorado, A. de Pablo, and U. Sánchez: A concave-convex elliptic problem involving the fractional Laplacian. - Proc. Roy. Soc. Edinburgh Sect. A 143:1, 2013, 39-71. 
[3] Bulut, H., T. A. Sulaiman, and B. Demirdag: Dynamics of soliton solutions in the chiral nonlinear Schrodinger equations. - Nonlinear Dynam. 91:3, 2018, 1985-1991.

[4] Chen, Q., H. Qin, J. Liu, J. Xiao, R. Zhang, Y. He, and Y. Wang: Canonical symplectic structure and structure-preserving geometric algorithms for Schrödinger-Maxwell systems. - J. Comput. Phys. 349, 2017, 441-452.

[5] Evanc, L. C.: Partial differential equation. - Amer. Math. Soc., 1998.

[6] Huang, Z., J. Xu, B. Sun, B. Wu, and X. Wu: A new solution of Schrödinger equation based on symplectic algorithm. - Comput. Math. Appl. 69, 2015, 1303-1312.

[7] Laskin, N.: Fractional Schröinger equations. - Phys. Rev. E 66:2, 2002, 056108.

[8] Lu, W.: Variational methods in partial differential equations. - Beijing Scientific Publishing House in China, 2002.

[9] Qiao, L.: Matsaev's type theorems for solutions of the stationary Schrödinger equation and its applications. - Discrete Contin. Dyn. Syst. 36:10, 2016, 5709-5720.

[10] Qiao, L.: Asymptotic behaviors of Green-Sch potentials at infinity and its applications. Discrete Contin. Dyn. Syst. Ser. B 22:6, 2017, 2321-2338.

[11] Qiao, L.: Solutions of the Dirichlet-Sch problem and asymptotic properties of solutions for the Schrödinger equation. - Appl. Math. Lett. 71, 2017, 44-50.

[12] Qiao, L., and G. PAN: Theorems of the Phragmén-Lindelöf type for subfunctions in a cone. - Proc. Edinb. Math. Soc. 60, 2017, 739-751.

[13] Qiao, L., and Y. REN: Integral representations for the solutions of infinite order of the stationary Schrödinger equation in a cone. - Monatsh. Math. 173:4, 2014, 593-603.

[14] Rabinowitz, P.: Minimax methods in critical point theory with applications to differential equations. - CBMS Reg. Conf. Ser. Math. 65, Amer. Math. Soc., Providence, RI, 1986.

[15] Rosenblum, G., M. Solomyak, and M. Shubin: Spectral theory of differential operators. VINITI, Moscow, 1989.

[16] Rudin, W.: Functional analysis. - China Machine Press, Beijing, second edition, 2004.

[17] Sasa, N., and J. Satsuma: New-type of soliton solutions for a higher-order nonlinear Schrödinger equation. - J. Phys. Soc. Japan 60:2, 1991, 409-417.

[18] Servadei, R., and E. Valdinoci: Variational methods for non-local operators of elliptic type. - Discrete Contin. Dyn. Syst. 33:5, 2013, 2105-2137.

[19] Shen, J., W. Sha, Z. Huang, M. Chen, and X. Wu: High-order symplectic FDTD scheme for solving a time-dependent Schrödinger equation. - Comput. Phys. Commun. 184:3, 2013, 480-492.

[20] Sulaiman, T. A., T. Akturk, H. Bulut, and H. M. Baskonus: Investigation of various soliton solutions to the Heisenberg ferromagnetic spin chain equation. - J. Electromagnetic wave $32: 9,2018,1093-1105$.

[21] Willem, M.: Minimax theorems. - Boston Birkhäuser Boston Inc, 1996.

[22] YoshidA, H.: Construction of higher order symplectic integrators. - Phys. Lett. A 150:5-7, 1990, 262-268.

[23] Zheng, C., and Z. Zhou: Exact controllability for the fourth order Schrödinger equation. Chin. Ann. Math. Ser. B 33:3, 2012, 395-404.

[24] Zhong, P., C. Zhang, and F. Wu: Energy decay rate of multidimensional inhomogeneous Landau-Lifshitz-Gilbert equation and Schrödinger map equation on the sphere. - Adv. Difference Equ. 2018, 2018, 335. 